\title{
Decreased choline acetyltransferase activity in the murine spinal cord motoneurons under chronic mechanical compression
}

\author{
Yoshiyuki Yato, Yoshikazu Fujimura, Masaya Nakamura, Masahiko Watanabe and Yutaka Yabe \\ Department of Orthopedic Surgery, School of Medicine, Keio University, 35 Shinanomachi, Shinjuku-ku, 160, Tokyo, \\ Japan
}

\begin{abstract}
The tiptoe-walking Yoshimura (twy) mouse is a model of chronic spinal cord compression caused by ossification of intraspinal ligaments. Choline acetyltransferase (CAT), which is known to be a specific marker of cholinergic neurons, best reflects spinal motoneuron function. Changes in CAT immunoreactivity following chronic spinal cord compression in twy mice were investigated quantitatively in order to elucidate spinal motoneuron functional changes according to the degree and direction of compression. Thirty 24-week-old twy mice were used in this study. They were divided into three groups according to the direction of spinal cord compression (anterior, posterior, and lateral) and the CAT immunoreactivities in whole sections of their upper cervical spinal cords were investigated quantitatively using a fluorescence microphotometry system. The lateral compression group showed histological spinal motoneuron atrophy and loss on the compressed, but not the non-compressed, side. Spinal motoneuron atrophy and loss were observed when the severity of spinal canal stenosis due to the ossified lesion, expressed as the occupation rate, was $30 \%$ or more, but the spinal motoneurons appeared normal when it was below $30 \%$. The CAT immunofluorescence intensity of the anterior horn showed a linear negative correlation with the degree of canal stenosis. When the occupation rate was below $20 \%$, the CAT immunofluorescence intensities in the anterior horns of the compression and control groups did not differ significantly. The CAT immunofluorescence intensity of twy mice with occupation rates of $20 \%$ or more were significantly lower than that of those with occupation rates below $20 \%$. Furthermore, the CAT immunofluorescence intensity was significantly lower on the compressed than the noncompressed side of the lateral compression group. Thus, our findings indicate that an occupation rate of about $20 \%$ may be the critical level for functional changes in the spinal motoneurons.
\end{abstract}

Keywords: cervical spinal cord; chronic compression; choline acetyltransferase; twy mouse

\section{Introduction}

Choline acetyltransferase (CAT), which is known to be a specific marker of cholinergic neurons, best reflects spinal motoneuron function. Although acetylcholine has been known as a neurotransmitter for many years, relatively little is known about the distribution or connections of spinal cholinergic neurons, ${ }^{1}$ mainly due to technical limitations to investigating cholinergic systems at the cellular level. The recent immunohistochemical method devised for detecting CAT provides a reliable means of localizing cholinergic neurons, ${ }^{2-6}$ and a new fluorescence microphotometry system has been developed which is extremely useful for analyzing the distribution and amount of neurotransmitters at the cellular level. ${ }^{7-9}$ This system can accurately measure the fluorescence intensity of CAT immunoreactivity,

Correspondence: Y Yato which provides a sensitive means of assessing the function of cholinergic neurons. ${ }^{10}$ It would therefore be very useful to apply these procedures to the investigation of CAT activity changes and distribution in the spinal cord of the mouse, because they should provide direct information about the gray matter. The aim of this study was to investigate CAT immunoreactivity changes following chronic spinal cord compression in mice in order to elucidate the functional changes in cholinergic neurons, especially motoneurons, according to the degree and direction of compression.

\section{Materials and methods}

Chronic spinal cord compression model (twy mouse) The tiptoe-walking Yoshimura (twy) mouse (Central Institute of Experimental Animals, Kawasaki, Japan) is a strain characterized by hereditary abnormalities of 
bone and cartilage. It exhibits contractures of the peripheral joints of its extremities and an abnormal gait described as 'walking tiptoe', 11 and develops chronic spinal cord compression due to ossification and/or calcification of the spinal ligaments, especially in the upper cervical region. ${ }^{12}$

In this study, 30 24-week-old twy mice were used as a model of chronic spinal cord compression (compression group) and 12 24-week-old Insitute of Cancer Research (ICR) mice were used as controls (control group).

\section{Immunohistochemistry}

Both the twy and ICR mice were perfused intracardially with $4 \%$ paraformaldehyde in phosphate-buffered saline (PBS), $\mathrm{pH} \mathrm{7.4,} \mathrm{their} \mathrm{cervical} \mathrm{spines} \mathrm{and} \mathrm{spinal}$ cords between $\mathrm{C} 1$ and $\mathrm{C} 3$ were removed en bloc, postfixed with the same solution for $48 \mathrm{~h}$, decalcified with $5 \%$ ethylenediaminetetraacetate for 7 days, and then cryoprotected by incubation with $10 \%$ buffered sucrose at $4{ }^{\circ} \mathrm{C}$ overnight. After quick freezing with dry ice and acetone, serial $10 \mu$ m-thick frozen sections were cut with a cryostat and stained immunohistochemically using the procedures reported previously. ${ }^{8,13}$ In brief, the sections were washed with PBS at $4{ }^{\circ} \mathrm{C}$, treated with anti-CAT rabbit serum, diluted $1: 100$ with PBS (Chemicon International, Temecula, CA, USA), for $12 \mathrm{~h}$ at $4^{\circ} \mathrm{C}$, washed with PBS, treated with FITClabeled anti-rabbit-IgG goat serum, ${ }^{14,15}$ diluted $1: 20$ with PBS (Tago; Biosourse International, Cararillo, CA, USA), for $2 \mathrm{~h}$ at room temperature and the stained sections were embedded in $20 \%$ glycerin-PBS.

\section{Fluorescence microphotometry}

The CAT immunofluorescence intensity of each anterior horn was measured using a microphotometry system (Model P-101; Nikon, Tokyo), ${ }^{10}$ which enables the average fluorescence intensity of a small spinal cord region to be measured through a measuring spot. The spinal cord section was moved in the $\mathrm{X}$ - and $\mathrm{Y}$ directions by means of a high-precision step-motor scanning stage under the objective lens of a fluorescence microscope and the fluorescence intensity of its entire surface was analyzed. The fluorescence intensity data and positions ( $\mathrm{X}$ and $\mathrm{Y}$ values) were transmitted to a PC9801FA computer (NEC, Tokyo), for statistical analysis. The microphotometry system conditions were: excitation range, $420-490 \mathrm{~nm}$; interference filter, $530 \mathrm{~nm}$; photo-multiplier voltage, $850 \mathrm{~V}$; objective lens, $20 \times / 0.75$ (magnification/aperture number) and measuring spot diameter, $40 \mu \mathrm{m}$. The mean fluorescence intensity of the anterior horn was determined by analyzing the data for the connected measuring points and the values presented are means \pm s.e.

\section{Histopathology}

After the CAT distribution analysis had been completed, the spinal cord sections were stained with hematoxylin-eosin and examined histologically under a light microscope. The twy mice were divided into three groups, according to the direction of spinal cord compression (anterior, posterior and lateral). If compression regions were present at more than one site, the group was assigned according to the direction of greatest compression. The degree of compression is expressed as the occupation rate $(\mathrm{A} / \mathrm{B} \times 100 \%$; $\mathrm{A}$ : area of ossified ligaments, $\mathrm{B}$ : cross-sectional area of the spinal canal).

\section{Statistical analysis}

Statistical significance was defined as $P<0.05$ using a non parametrictest followed by the Mann-Whitney Utest.

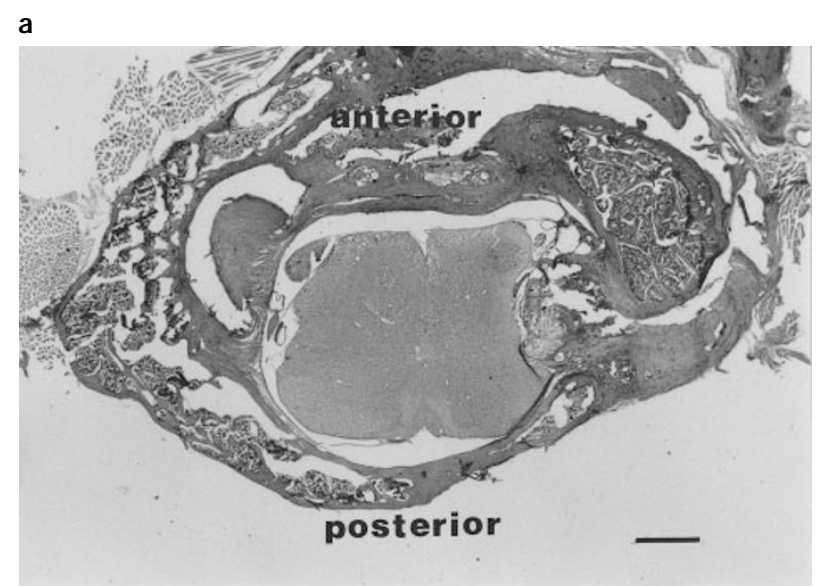

b

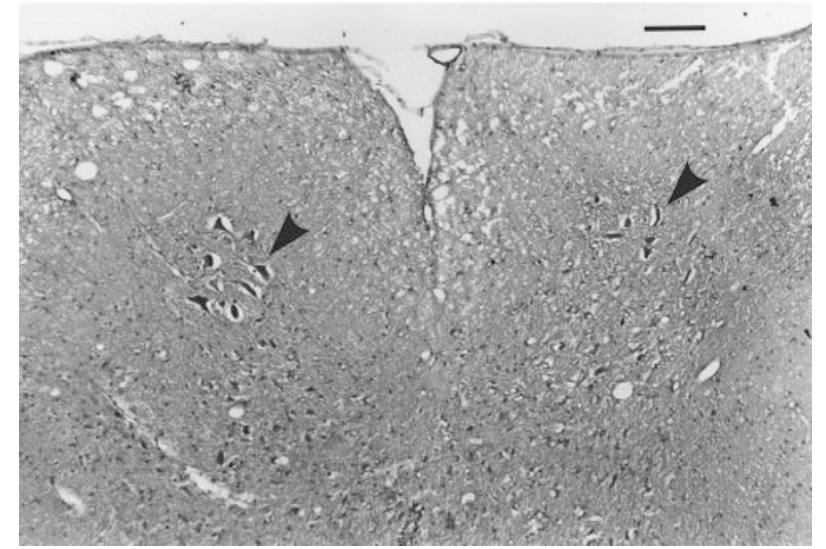

Figure 1 (a) The lateral compression group. (Hematoxylineosin staining. Scale bar: $1000 \mu \mathrm{m}$ ). (b) Atrophy and loss of the anterior horn cells were observed on the compressed side, but not on the non-compressed side. (Hematoxylin-eosin staining. Scale bar: $100 \mu \mathrm{m})$ 


\section{Results}

\section{Histopathological findings}

No histological changes were observed in the anterior horn cells of the control group. There were eight, six and 16 twy mice in the anterior, posterior and lateral compression groups respectively. In the lateral compression group, anterior horn cell atrophy and loss were observed on the compressed, but not on the noncompressed, side. There were no histological differences between the right and left sides of either the anterior or posterior compression groups (Figure 1). There were no histopathological changes in the anterior horn cells of the twy mice, with occupation rates below $20 \%$ (mild compression). Anterior horn cell loss and atrophy were observed in those with occupation rates of $30 \%$ or more (severe compression), and those with occupation rates of $20-30 \%$ (moderate compression), showed few histopathologic changes in the anterior horn cells (Figures 2, 3, 4 and Table 1).

a

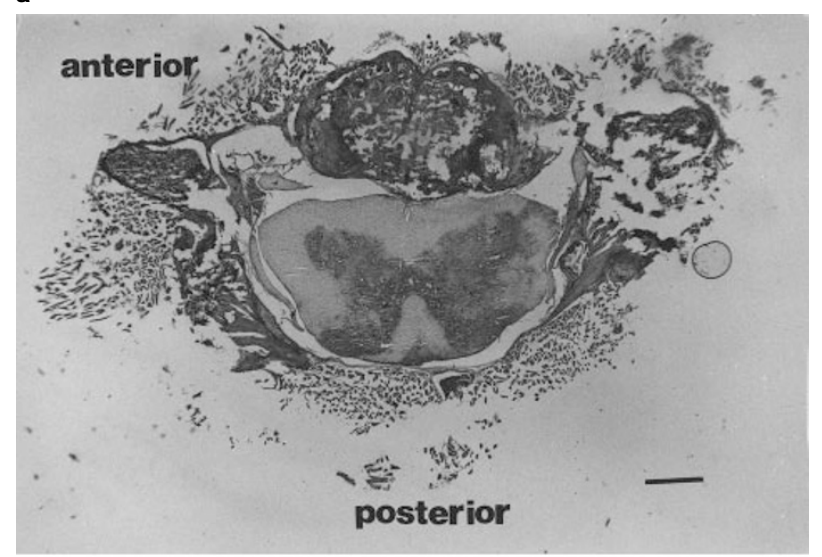

b

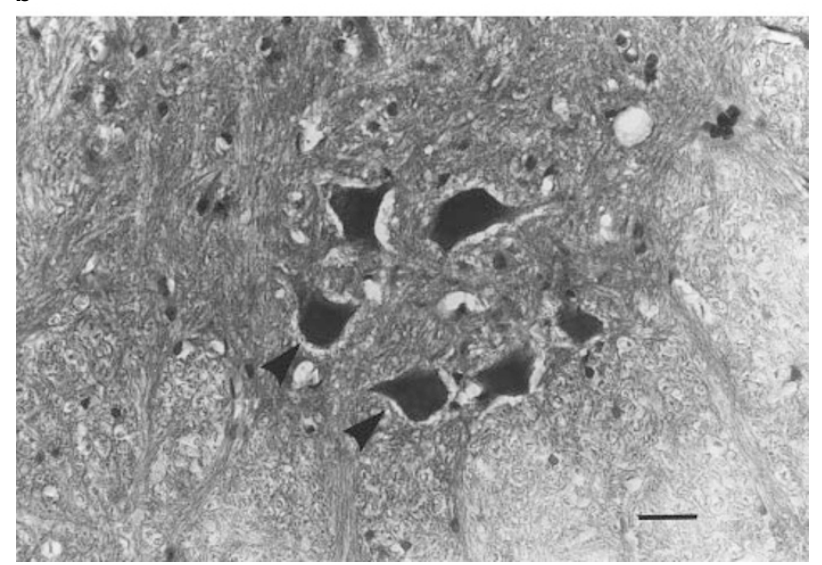

Figure 2 (a) The mild compression was observed in the spinal cord. (occupation rate $<20 \%$ ) (Hematoxylin-eosin staining. Scale bar: $1000 \mu \mathrm{m}$ ). (b) There were no histopathological changes of the anterior horn cells. (Hematoxylin-eosin staining. Scale bar: $100 \mu \mathrm{m}$ )
CAT immunofluorescence intensity changes in the anterior horn

CAT-immunoreactive neurons were present in the ventrolateral anterior horns of the ICR and twy mice, and the CAT immunofluorescence intensity in

Table 1 Occupation rate and histological changes of anterior horn cells

\begin{tabular}{lcc}
\hline Occupation rate & Cell atrophy & Cell loss \\
\hline$\sim 20 \%$ & $1 / 13$ & $0 / 13$ \\
$20 \sim 30 \%$ & $4 / 13$ & $2 / 13$ \\
$30 \% \sim$ & $4 / 4$ & $4 / 4$ \\
\hline
\end{tabular}

There were no histopathological changes in the anterior horn cells at occupation rates of less than 20\%. Atrophy and loss of anterior horn cells were observed at occupation rates of $30 \%$ or more. On the other hand, at occupation rates of $20-$ $30 \%$, histopathological changes in the anterior horn cells were observed in four mice out of 13

a

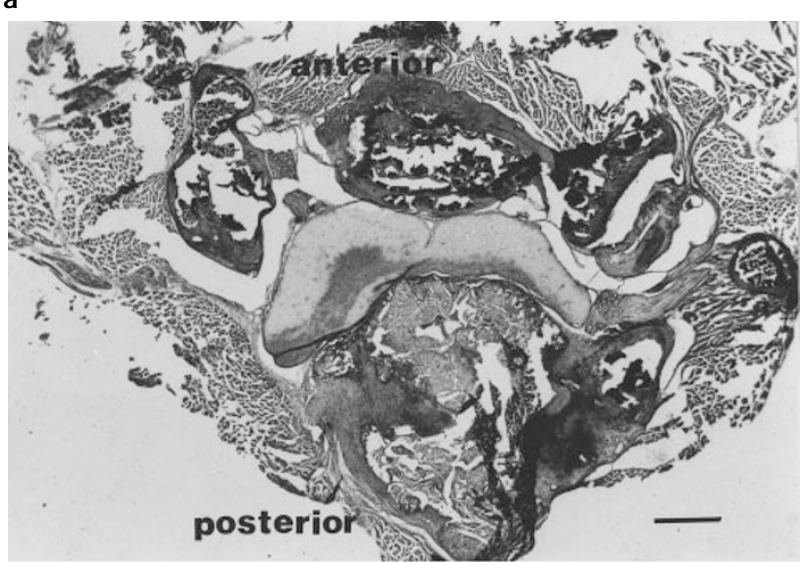

b

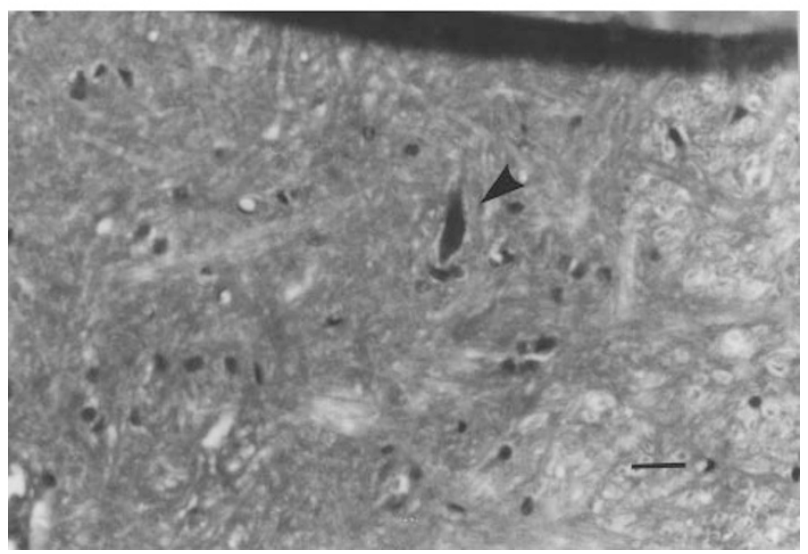

Figure 3 (a) The severe compression was observed in the spinal cord. (occupation rate $>30 \%$ ) (Hematoxylin-eosin staining. Scale bar: $1000 \mu \mathrm{m}$ ). (b) Atrophy and loss of the anterior horn cells were observed. (Hematoxylin-eosin staining. Scale bar: $100 \mu \mathrm{m})$ 
the anterior horn showed a linear negative correlation with the occupation rate (Figure 5). Moreover, the CAT immunofluorescence intensity of the anterior horn in twy mice with occupation rates below $20 \%$ (mild compression), did not differ significantly from that of the control group. The CAT immunofluorescence intensities of mice with occupation rates of $20 \%$ above (moderate and severe compression) were significantly lower than that of those with occupation rates below 20\% (mild compression) (Figure 6). Furthermore, in the lateral compression group, the CAT immunofluorescence intensity on the compressed side was significantly lower than that on the noncompressed side (Figure 7).

\section{Discussion}

It is well known that severe chronic spinal cord compression causes histological changes, such as

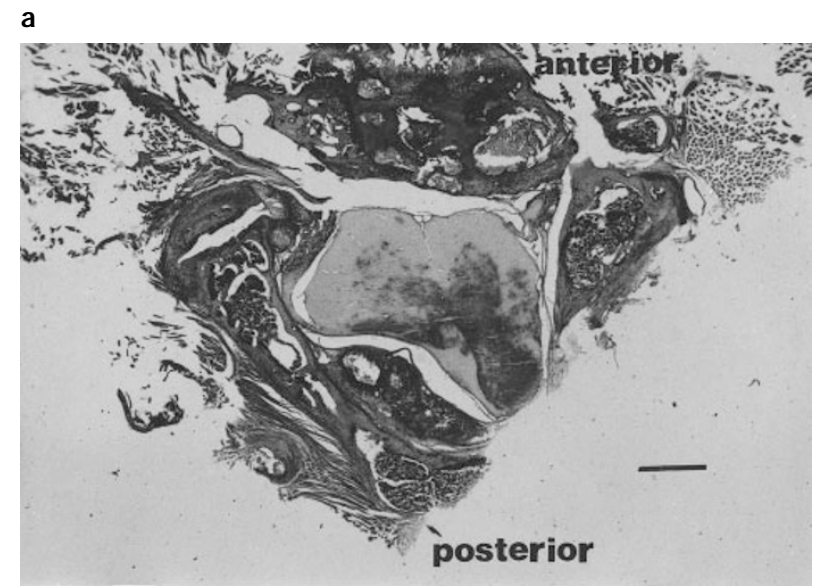

b

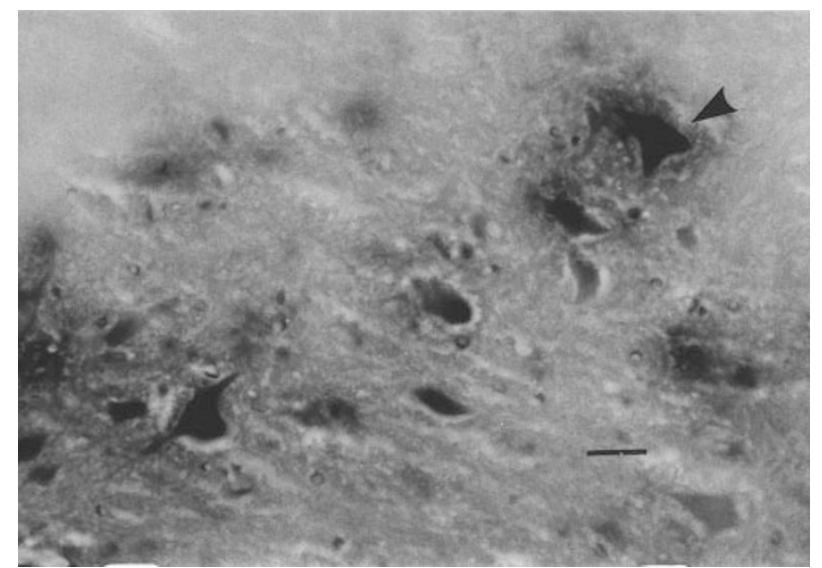

Figure 4 (a) The moderate compression was observed in the spinal cord. (occupation rate 20-30\%) (Hematoxylin-eosin staining. Scale bar: $1000 \mu \mathrm{m})$. (b) There was atrophy of the anterior horn cells, but the number of the anterior horn cells was kept normally. (Hematoxylin-eosin staining. Scale bar: $100 \mu \mathrm{m})$

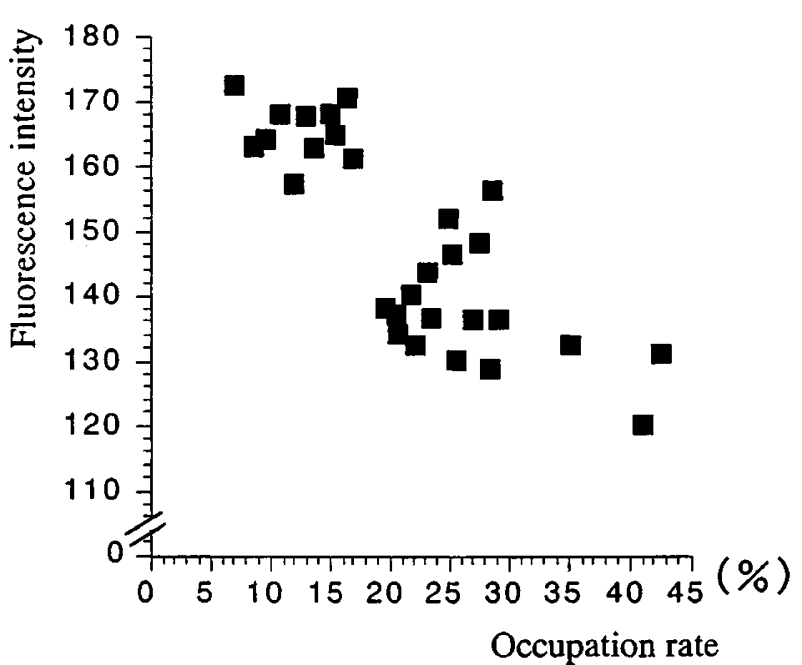

Figure 5 CAT immunofluorescence intensity in the anterior horn showed a linear negative correlation with occupation rates $(r=0.839, P<0.0001)$

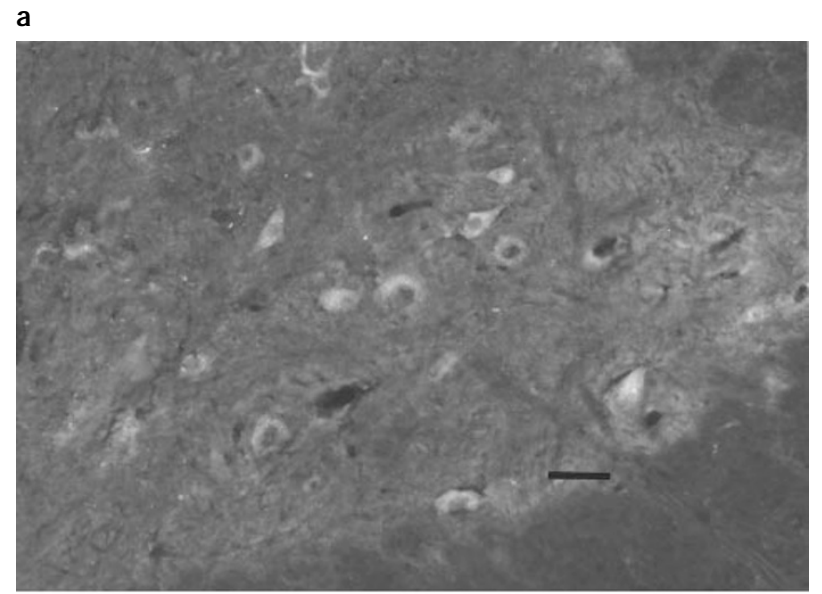

b

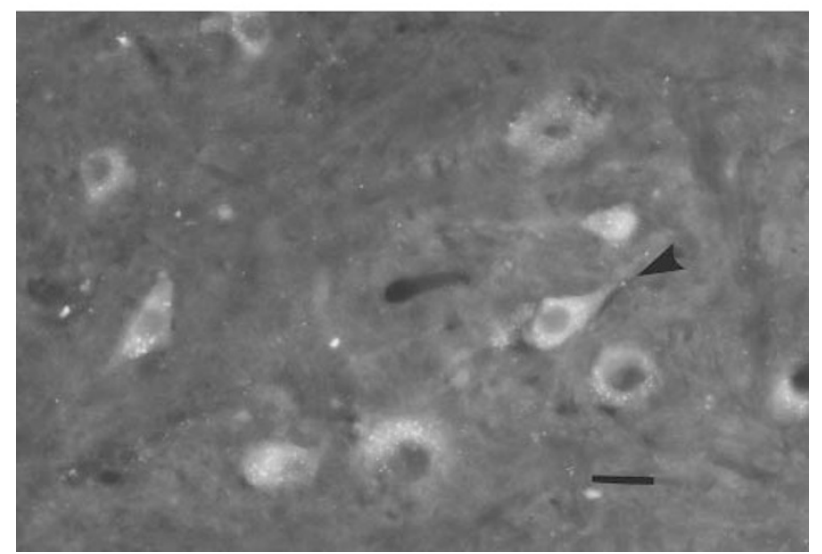

Figure 6 (a) CAT immunofluorescence microphotometory of the anterior horn. Control group. (Scale bar: $200 \mu \mathrm{m}$ ). b) CAT immunofluorescence microphotometory of the anterior horn cells. Control group (Scale bar: $100 \mu \mathrm{m}$ ) 
anterior horn cell atrophy and loss. ${ }^{16,17}$ However, exactly what degree of chronic spinal cord compression causes functional changes in cholinergic neurons, especially spinal motoneurons, is unclear. One reason for this is that functional changes in the gray matter, especially of spinal motoneurons, could not be demonstrated, due to difficulty actually reaching the gray matter and elucidating its functions. In this study, we used a new fluorescence detection system to overcome this problem and demonstrated that the CAT immunoreactivity changes correlated with the severity of the histopathologic changes in the chronically compressed spinal cord. Another reason was the lack of availability of a satisfactory chronic spinal compression model. Although many animal models of chronic spinal cord compression have been described, most of them have proved artificial and unsatisfactory. ${ }^{1}$ The twy mouse exhibits multi-level spinal canal stenosis resulting from the natural occurrence of ossification of various spinal ligaments, including those of the upper cervical spine. ${ }^{11,12}$ Therefore, we chose this animal as the chronic spinal compression model for use in our study.

We found that mild chronic spinal cord compression (occupation rate $<20 \%$ ) caused neither histopathologic nor CAT immunoreactivity changes, whereas severe chronic spinal cord compression (occupation rate $>30 \%$ ) caused spinal motoneuron atrophy and/or loss and reduced CAT immunoreactivity, in the anterior horn. Some investigators showed that the transverse area was correlated with the severity of pathological changes ${ }^{16}$ and that chronic compressed spinal cord with a transverse area below $60 \%$ of normal appeared to be associated with severe and irreversible pathological changes in patients with ossification of the posterior longitudinal ligament, ${ }^{18}$ which is consistent with our findings. Furthermore, we found that moderate chronic spinal cord compression $(20 \%<$ occupation rate $<30 \%$ ) did not cause histologic changes, but resulted in a significant decrease in the CAT immunoreactivity in the anterior horn. In other words, moderate chronic spinal cord compression caused functional changes in the spinal motoneurons

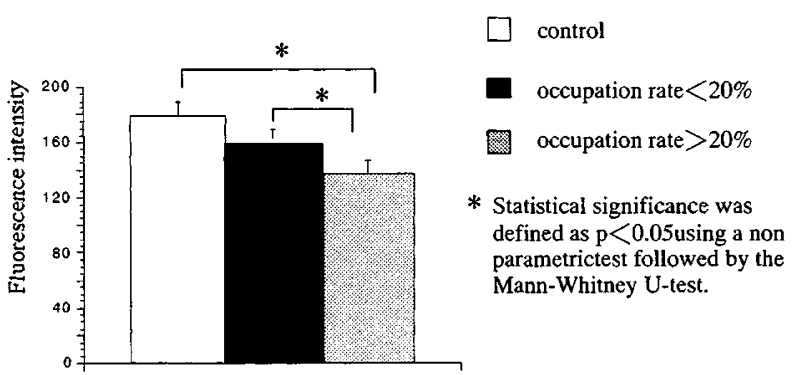

Figure 7 CAT immunofluorescence intensity at occupation rates of $20 \%$ and above were significantly lower than at occupation rates of less than $20 \%$ prior to the development of histopathologic changes. These findings indicate that moderate chronic spinal cord compression (occupation rate of around 20\%) may be the critical level for functional changes in spinal motoneurons.

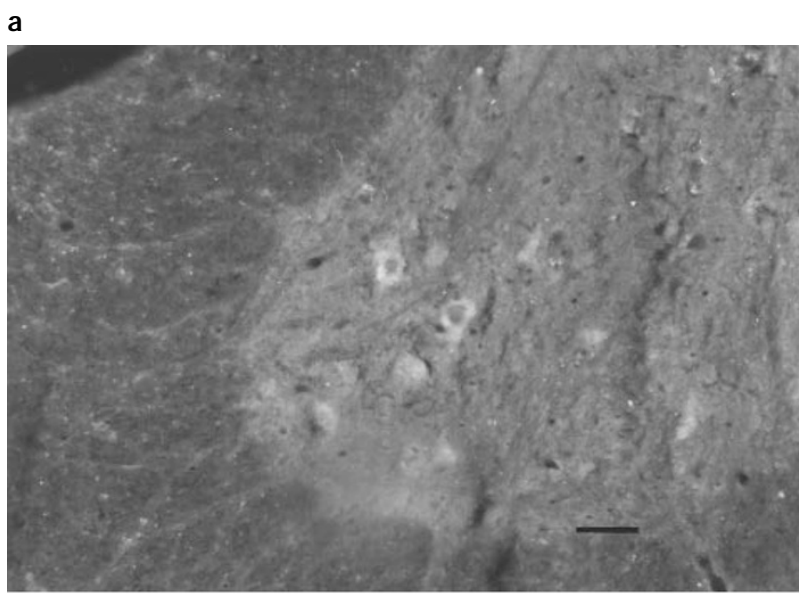

b

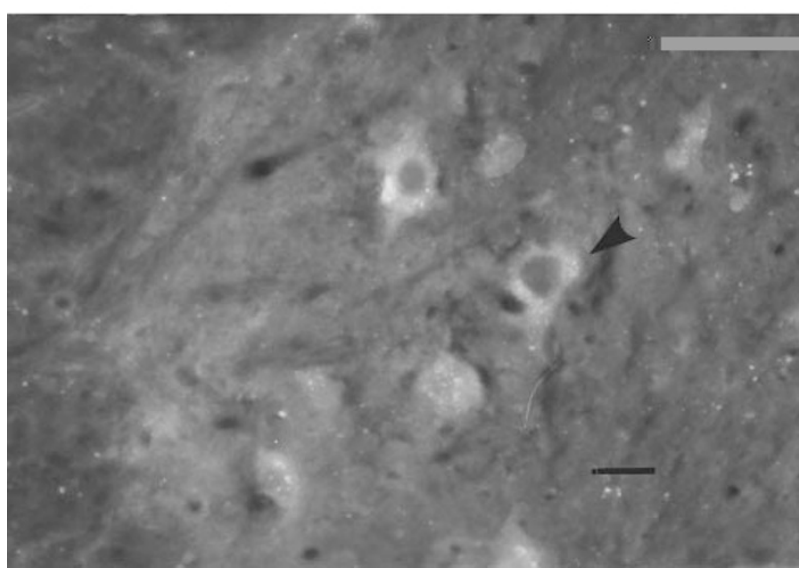

Figure 8 (a) CAT immunofluorescence microphotometry of the anterior horn. Moderate compression group. (Scale bar: $200 \mu \mathrm{m}$ ). (b) CAT immunofluorescence microphotometry of the anterior horn cells. Moderate compression group. (Scale bar: $100 \mu \mathrm{m})$

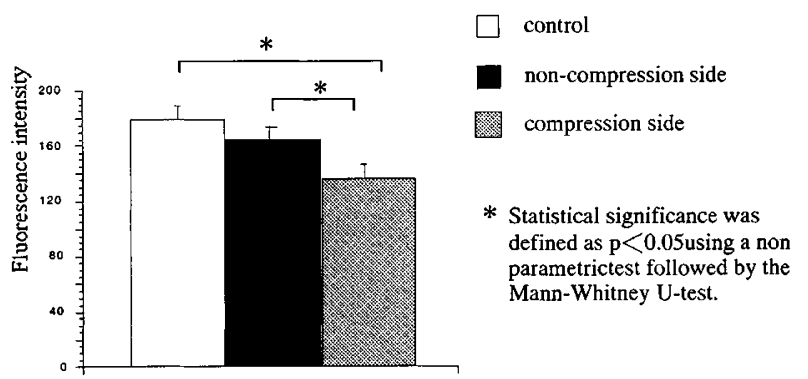

Figure 9 In the lateral compression group, CAT immunofluorescence intensity on the compressed side was significantly lower than on the non-compressed side 


\section{References}

1 Arbit E, Galicich W, Galicich JH, Lau N. An animal model of epidural compression of the spinal cord. Neurosurgery 1989; 24: $860-863$.

2 Borges LF, Iversen SD. Topography of choline acetyltransferase immunoreactive neurons and fibers in the rat spinal cord. Brain Res 1986; 362: $140-148$.

3 Crawford GD, Salvaterra PM, Vaughn JE. The morphology and distribution of neurons containing choline acetyltransferase in the adult rat spinal cord; an immunohistochemical study. J Comp Neurol 1984; 229: $329-346$

4 Eckenstein F, Thoensen H. Cholinergic neurons in the rat cervical cortex demonstrated by immunohistochemical localization of choline acetyltransferase. Neurosci Lett 1983; 36: $211-215$.

5 Houser CR et al. Organization and morphological characteristics of cholinergic neurons. Brain Res 1983; 266: 97-119.

6 Kimura H, McGeer PL, Peng JH, Mcgeer EG. The central cholinergic system studied by choline acetyltransferase immunohistochemistry in the cat. J Comp Neurol 1981; 200: 151-201.

7 Sutoo D, Akiyama K, Geltard M. Central dopamine-synthesis regulation by the calcium-calmodulin-dependent system. Brain Res Bull 1989; 22: $565-569$.

8 Sutoo D, Akiyama K, Imaniya S. A mechanism of cadmium poisoning: the cross-effect of calcium and cadmium in the calmodulin-dependent system. Arch Toxlcol 1990; 64: 161-164.

9 Sutoo D, Akiyama K, Yabe K, Nishida N. Quantitative immunohistochemical distribution of choline acetyltransferase in the rostral forebrain of the rat. Neurosci Res 1991; 11: 63-70.

10 Sutoo D, Akiyama K, Maeda I. The development of high sensitivity and high linearity fluorescence microphotometry system for distribution analysis of neurotransmitter in the brain. Nippon Yakurigaku Zassi (Folia Pharmacol Japan) 1988; 91: $173-180$.

11 Hosoda Y, Yoshimura Y, Higashi S. A new breed of mouse showing multiple osteochondral lesions -twy mouse-. Ryumachi 1981; 21: $157-164$.

12 Hosoda Y et al. A morphological study of upper cervical spines of twy mouse. Connect Tissue 1989; 21: 7 -8.

13 Nakamura $\mathrm{M}$ et al. Changes in choline acetyltransferase activity and distribution following incomplete cervical spinal cord injury in the rat. Neuroscience 1996; 75: $481-494$.

14 Armstrong DM, Bruce G, Hersh IB, Terry RD. Cholineacetyltransferase immunoreactivity in neuritic plaques of Alzheimer brain. Neurosci Lett 1986; 71: 229-234.

15 Bruce G, Wainer BH, Hersh LB. Immunoaffinity purification of human choline acetyltransferase: comparison of the brain and placental enzymes. $J$ Neurochem 1985; 45: $611-620$.

16 Fujiwara $\mathrm{K}$ et al. Morphometry of the cervical spinal cord and its relation to pathology in cases with compression myelopathy. Spine 1988; 13: $1212-1216$.

17 Ono K, Tada K, Yamamoto T. Cervical myelopathy secondary to multiple spondylotic protrusions: a clinicopathologic study. Spine 1977; 2: $109-125$.

18 Kameyama $\mathrm{T}$ et al. Spinal cord morphology and pathology in ossification of the posterior longitudinal ligament. Brain 1995; 118: $263-278$ 